\title{
Tratamento farmacológico e interações medicamentosas em pacientes com aneurisma da aorta abdominal
}

\section{Pharmacological treatment and drug interactions in patients with abdominal aortic aneurysm}

Recebido em: 18/02/2015 Aceito em: 28/04/2015
Heverton Alves Peres ${ }^{1}$, Sergio Henrique Rafaine de Castro ${ }^{2}$

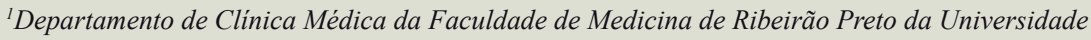
de São Paulo (USP), Av. Bandeirantes, 3900, CEP 14049-900 Ribeirão Preto, SP, Brasil. ${ }^{2}$ Universidade de Franca, Av Doutor Armando de Sales Oliveira, 201- Parque Universitário, CEP 14404-600,São Paulo,Brasil.E-mail: haperes@usp.br

\begin{abstract}
The abdominal aortic aneurysm (AAA) occurs when there is an abnormal and irreversible dilation of the artery, greater than $50 \%$ of the size expected for the vessel. It is associated with a mortality rate of 80 to $90 \%$. The control of the main risk factors such as hypertension, dyslipidemia, smoking and thrombotic diseases in small aneurysms is done by several drugs that prevent the development of aneurysm and rupture, however, the use of various medications associated with significant drug interactions can initiate and crucial knowledge about these. Currently, there are few data in the literature about the treatment and drug interactions in patients with AAA, and the purpose of this review is to describe pharmacotherapy and the interactions drugs in patients with AAA.
\end{abstract}

Keywords: abdominal aortic aneurysm, drug-drug interaction; pharmacotherapy

\section{RESUMO}

O aneurisma da aorta abdominal (AAA) ocorre quando há uma dilatação anormal e irreversível da artéria superior a $50 \%$ do calibre esperado para o vaso e associa-se a uma mortalidade de 80 a $90 \%$. O controle dos principais fatores de risco como a hipertensão, dislipidemia, tabagismo e doenças trombóticas em aneurismas pequenos é feito por vários medicamentos que evitam o desenvolvimento e ruptura do aneurisma, no entanto, o uso destes associados a outros medicamentos pode desencadear interações medicamentosas relevantes sendo crucial o conhecimento sobre estas. Atualmente, há poucos dados na literatura sobre o tratamento farmacológico e interações medicamentosas em pacientes com AAA, sendo o objetivo desta revisão, descrever a farmacoterapia e interações medicamentosas em pacientes com AAA.

Palavras chave: aneurisma da aorta abdominal, interações medicamentosas, farmacoterapia 


\section{INTRODUÇÃO}

A palavra aneurisma é derivada do vocabulário grego aneurysma que significa dilatação, e corresponde a um vaso sanguíneo com $50 \%$ de diâmetro maior que o normal. $\mathrm{O}$ aneurisma da aorta abdominal (AAA) ocorre quando há uma dilatação anormal e irreversível da artéria e associa-se a uma mortalidade de 80 a $90 \%$ devido ao rompimento da artéria, que é a complicação pré-hospitalar mais grave $(1,2)$.

Os dados da literatura determinam diferentes valores na prevalência do AAA devido às características clínicas de cada amostra e região. No Brasil um estudo retrospectivo feito de 1998 a 2007 com dados do registro de internações e procedimentos do Sistema Único de Saúde, revelou que o AAA e os aneurismas ocupam a $30^{\circ}$ causa mais frequente de óbito na população do Estado de São Paulo, sendo 3.539 óbitos de homens devido à dissecação da aorta e 1.383 óbitos referentes à ruptura do AAA (3). A prevalência do AAA é 4\% a $8 \%$ em homens e em mulheres $0,5 \%-1,5 \%$, variando de acordo com raça, idade, história familiar, localização geográfica e consumo de cigarro (4).

Ainda no Brasil, um estudo transversal no período de 2008-2009, realizado pelo Serviço de Cirurgia Vascular do Hospital Geral Roberto Santos em Salvador (Bahia), selecionou 1350 indivíduos com 50 anos ou mais que apresentavam fatores de risco para AAA, e os resultados deste estudo, demonstraram uma prevalência de AAA de 3,9\% com predomínio em homens na proporção $3: 1$. Neste mesmo estudo, quando analisado somente o grupo dos homens, a prevalência foi de $8,9 \%$. Este fato ocorreu devido ao fato de os pacientes do sexo masculino apresentarem maiores números de fatores de risco para doenças cardiovasculares. Os fatores de risco mais frequentes associados à AAA neste estudo foram: gênero masculino, tabagismo, antecedente de AAA e portadores de doença arterial oclusiva periférica, insuficiência coronariana e doença pulmonar obstrutiva crônica (5). Nos Estados Unidos da América (EUA), a doença é $13^{\text {a }}$ causa de óbitos sendo responsável por 30.000 óbitos por ano, principalmente em indivíduos com mais de 60 anos (1-6).

O diagnóstico do AAA é clínico com manifestações inespecíficas (edema nos membros inferiores, dores lombar ou abdominal, entre outros) e/ou por meio de exames de imagem. Em alguns casos, o diagnóstico é feito quando os sintomas e sinais já se manifestaram como: eventos tromboembólicos isquêmicos, ruptura e compressão das estruturas adjacentes. Os métodos que auxiliam no diagnóstico do AAA são: radiografia de co- luna lombar, ultrassonografia, tomografia computadorizada abdominal e angioressonância magnética $(6,7)$.

Vários avanços significativos nos meios de diagnósticos e técnicas cirúrgicas ocorreram nos últimos 50 anos (8), porém não foi verificada uma melhora significativa da mortalidade associada à ruptura do AAA (9). Os avanços nos meios de diagnósticos, a introdução dos programas de rastreio associado ao aumento da expectativa e o aumento do número de fumantes nas duas últimas décadas, têm contribuído para o a incidência do AAA (9-10).

Atualmente, não existe um "padrão ouro" no tratamento farmacológico do AAA e este tem sido motivo de constantes discussões, pois poucos alvos terapêuticos foram validados em humanos até o presente momento (10). No AAA de pequeno tamanho $(<5,5)$ com crescimento menor que $0,5 \mathrm{~cm}$ em seis meses e assintomático, há uma baixa taxa de ruptura; porém o acompanhamento dos pacientes é semestral e o manejo do aneurisma não requer cirurgia podendo ser controlado com medicamentos anti-hipertensivos, antilipêmicos, antiplaquetários e a cessação do tabagismo para evitar a ruptura do aneurisma. O objetivo do tratamento farmacológico é evitar a dilatação subjacente, o rompimento da aorta e controlar os fatores de risco descritos acima.

A Atenção Farmacêutica envolve o acompanhamento do paciente com dois objetivos principais sendo: responsabiliza-se junto com o paciente para que o medicamento prescrito pelo médico seja seguro e eficaz em posologia na dosagem correta, resultando em efeito terapêutico desejado; certificar-se que ao longo do tratamento, as reações adversas aos medicamentos sejam mínimas e quando surgirem, que possam ser resolvidas imediatamente pelo farmacêutico. O foco da Atenção Farmacêutica é o paciente, sendo importante estabelecer um elo de confiança entre este e o farmacêutico, visando uma farmacoterapia racional para melhorar a qualidade de vida do paciente $(12,13)$. Os pacientes com AAA fazem uso de diversas classes de fármacos, aumentando as chances de interações medicamentosas, e devido a este fato, o acompanhamento farmacoterapêutico é imprescindível.

Atualmente, poucos estudos clínicos randomizados avaliaram a eficácia dos medicamentos usados no AAA $(14,15,16)$. A farmacoterapia atual usada no tratamento do AAA constitui-se de betabloqueadores (Propranolol), antagonistas dos receptores de angiotensina (losartana), inibidores da ECA (captopril) estatinas (sinvastatina) e antiplaquetários (ticlopidina) (23-24). Neste contexto, sabendo que a maioria dos pacientes com AAA tem idade mais avançada e faz uso várias classes 
farmacológicas, aumentando as chances reais de interações medicamentosas, devido às várias limitações fisiológicas que influenciam na farmacocinética, o objetivo do presente estudo, é discutir a farmacoterapia e interações medicamentosas em pacientes com AAA.

\section{MÉTODO}

Os dados coletados para o presente estudo foram obtidos em livros e bases de dados eletrônicas como Science Direct, Pubmed e Scielo publicados entre 2000 e 2015. Para tal pesquisa foram utilizados, de modo integrado, descritores como aneurisma da aorta abdominal, interações medicamentosas e farmacoterapia.

\section{RESULTADOS}

O presente estudo é o primeiro no Brasil a revisar a literatura e discutir as interações medicamentosas em pacientes com AAA. Na farmacoterapia do AAA, o objetivo é controlar os fatores de risco que contribuem para o desenvolvimento do AAA, tais como a hipertensão, hipercolesterolemia, doenças tromboembolíticas e controle do tabaco (18-19). No controle da pressão arterial (PA) as principais drogas usadas nos pacientes com AAA são: propranolol, losartana e captopril.

O propranolol é um fármaco que pertence à classe dos beta-bloqueadores alfa-adrenérgicos e atua no sistema cardiovascular inibindo as respostas cronotrópicas, inotrópicas e vasoconstritoras à ação das catecolaminas epinefrina e norepinefrina nos receptores beta-adrenérgicos. Nos últimos 40 anos, os beta-bloqueadores têm demonstrado eficácia na redução da pressão arterial, e hoje constituem a primeira opção terapêutica na hipertensão arterial associada à doença coronária, às arritmias cardíacas, à enxaqueca, entre outras indicações compulsórias (20-21). O mecanismo exato para a redução da pressão arterial e controle da AAA é complexo e ainda não está elucidado, mas alguns efeitos têm sido propostos. O bloqueio dos receptores $\beta 1$ adrenérgicos cardíacos causa redução da frequência cardíaca e da contratilidade, com a consequente redução do débito cardíaco, enquanto a ação nas células justaglomerulares renais diminui a liberação de renina (22-23). Também existem relatos de que os beta-bloqueadores promovem readaptação dos barorreceptores e diminuição das catecolaminas nas sinapses nervosas. A dose do propranolol é diferente para cada indicação e orientação médica; entretanto, para hipertensão. a dose geralmente é de $40 \mathrm{mg}$, duas vezes ao dia, de preferência após a refeição, pois os alimentos reduzem o metabolismo do propranolol e aumentam a disponibilidade biológica e eficácia desse fármaco (22-23).

A losartana potássica é um fármaco que pertence à classe dos antagonistas dos receptores da angiotensina, BRA, sendo eficaz no controle da pressão arterial e na redução da morbimortalidade pelas doenças cardiovasculares. O mecanismo de ação se deve ao antagonismo dos receptores $\mathrm{AT}^{1}$ da angiotensina, que promovem vasodilatação direta e impedem o aumento da aldosterona pelas glândulas suprarenais, diminuindo a retenção de sódio e água evitando a hipervolemia. A combinação de um BRA e um diurético aumenta a eficácia sobre o bloqueio do receptor $\mathrm{AT}^{1}$, devido ao fato do diurético estimular a atividade plasmática da renina, sendo que a pressão arterial se torna mais sensível à depleção de volume induzida pelo diurético. A dose de losartana é de 25-100 mg uma vez ao dia, com ou sem alimentos. Todavia, a ingestão concomitante, moderada ou alta, de potássio pode levar a hipercalemia por aumento dos níveis séricos de potássio devido à inibição na secreção de aldosterona induzida pela angiotensina II (22-24). A ingestão do suco de toranja junto com a losartana influi na formação do principal metabólito, o E3174. O provável mecanismo desta interação é a inibição do CYP3A4 em sua primeira passagem pela parede intestinal devido à presença dos flavonoides naringina e naringenina e furanocumarinas (bergamotina) do suco de toranja. A relevância clínica desta interação ainda é desconhecida, no entanto, a frequência e a quantidade de suco de toranja ingerida por pacientes em uso da losartana, devem ser monitoradas (25). Os pacientes com diabetes, insuficiência cardíaca e/ou renal estão sob maior risco de desenvolver hipercalemia e os níveis de potássio devem ser monitorados.

O captopril pertence ao grupo dos inibidores da enzima conversora de angiotensina (IECA) e o desenvolvimento deste grupo iniciou-se com a descoberta de um fator potencializador da bradicinina que, em seguida, demonstrava que esse novo grupo inibia a conversão enzimática da angiotensina I em angiotensina II (26-27). Os IECA são bem tolerados na maioria dos pacientes, sendo a tosse seca e a hipercalemia como efeitos adversos mais relatados. Entretanto, nessa situação, a substituição do IECA por um BRA pode ser uma opção. Um efeito clássico dos IECA é diminuição da taxa de filtração glomerular que atenua a função renal principalmente em pacientes com desidratação, insuficiência cardíaca e em uso de AINE. Isto ocorre devido à diminuição do fluxo aferente da artéria renal que reduz de forma temporária a filtração glomerular e, devido a isso, há um aumento da produção de angiotensina II e em resposta a esse quadro, ocorre constrição da arteríola eferente para 
restabelecer a pressão hidrostática no leito capilar glomerular (22-28).

A dose de captopril é 25-75 mg ao dia, uma hora antes ou duas horas após as refeições, pois os alimentos reduzem sua biodisponibilidade (29-30). Os diuréticos poupadores de potássio (espironolactona, amilorida, triantereno e eplerenona) associados aos IECA podem produzir hipercalemia, principalmente em idosos, diabéticos e pacientes com doença renal, sendo necessária a introdução de diurético de alça ou tiazídico (30-31). Os AINE associados aos IECA favorecem o desenvolvimento de hipercalemia devido a deterioração da função renal e os AINE atenuam na liberação de renina mediada pelas prostaglandinas, reduzindo a formação de aldosterona e em consequência a diminuição de potássio. Os AINE reduzem os efeitos dos anti-hipertensivos por interferir na síntese de prostaglandinas renais que são responsáveis pela vasodilatação, redução da resistência vascular renal e aumento a perfusão do órgão, sendo que a inibição destes processos culmina vasoconstrição renal e aumento da pressão arterial (31).

Para redução de trombos e coágulos em pacientes com AAA, o principal fármaco utilizado é a ticlopidina um antiplaquetário usado no tratamento e prevenção da trombose artéria, produzindo inibição da agregação das plaquetas e da liberação de fatores plaquetários, sendo esta inibição dependente da dose e do tempo de administração (32). A ticlopidina é direcionada a pacientes com trombos e coágulos que obstruem as artérias e veias podendo causar um aneurisma, infarto do miocárdio e acidentes cardiovasculares. Os pacientes com uso da ticlopidina devem fazer um acompanhamento severo por meio de hemogramas completos, devido à diminuição do número de plaquetas da corrente sanguínea. A dose de ticlopidina é $250 \mathrm{mg} /$ dia sendo administrado após as refeições. O uso concomitante de ticlopidina e ácido acetilsalicílico (AAS) ou varfarina não é aconselhado devido ao risco de hemorragias. O mecanismo dessa interação ocorre devido ao aumento da atividade anti-agregante plaquetária, produzindo lesão à mucosa gástrica. Caso seja necessário alguma dessas associações, é necessário um controle clínico laboratorial do paciente.

Para reduzir os níveis de colesterol e evitar a formação de placas lipídicas que se depositam nas paredes das artérias, os pacientes com AAA fazem uso da sinvastatina. $\mathrm{O}$ uso da sinvastatina é recomendado a estes pacientes devido à redução de mortes por doenças cardiovasculares (33). A sinvastatina é um fármaco pertencente à classe das estatinas que atua por competição da enzima hidroximetilglutarilcoenzima A redutase (HMGCoA-redutase), a qual está envolvida na cadeia de reações químicas, que ocorrem para a síntese de colesterol.
Com a redução da quantidade de colesterol formado no hepatócito, ocorre maior síntese de receptores de membrana que captam lipoproteínas ricas em colesterol as quais se reduzem na circulação. A dose de sinvastatina pode variar de 5 a $80 \mathrm{mg}$ ao dia, sendo tomada à noite, pois a síntese de colesterol ocorre à noite e as concentrações do colesterol total e do LDL colesterol aumentam significativamente quando o fármaco é usado pela manhã. O grupo da estatinas apresenta outras atividades farmacológicas além da atividade antilipêmica como: anti-inflamatória, melhora da função endotelial, antioxidante, redução da adesividade plaquetária e formação de trombos (34-35). Entre os efeitos adversos mais severos, destacam-se a miopatia e rabdomiólise ou necrose muscular. O risco de rabdomiólise é aumentado pelo uso concomitante de sinvastatina e fármacos com efeito inibitório significativo na isoforma $3 \mathrm{~A} 4$ do citocromo P450 em doses terapêuticas (ciclosporina, mibefradil, itraconazol, cetoconazol, eritromicina, claritromicina, inibidores da protease do HIV e nefazodona) ou com derivados do ácido fíbrico (genfibrozila) ou niacina. Se utilizada concomitantemente com ciclosporina, genfibrozila, outros fibratos (exceto fenofibrato) ou doses hipolipemiantes de niacina, a dose de sinvastatina não deve ser maior do que $10 \mathrm{mg} /$ dia (36). Estudos realizados por Sugimoto et al. (2001) revelaram que a utilização concomitante de Hyperico perforatum (erva de são-joão, hipérico) e sinvastatina em voluntários sadios reduz os níveis plasmáticos da sinvastatina em até $52 \%$ por inibição do CYP3A4 (37).

Novos alvos terapêuticos têm sido propostos para combater o AAA. As interleucinas IL-6 e IL- $\beta$ apresentam papel crucial na inflamação que ocorre no desenvolvimento do AAA (38-39). Uma revisão sistemática e uma meta-análise dos estudos com níveis circulantes de IL-6 revelaram que os pacientes com AAA têm níveis circulantes de IL-6 superiores comparados ao controle, proporcionando evidências de que a variante funcional (Asp358Ala) do gene da IL-6 está associada com o desenvolvimento do AAA, demonstrando que a IL-6 pode ser um novo alvo terapêutico. No entanto, estudos clínicos controlados precisam ser feitos para comprovar essa hipótese (38). Em relação a IL- $\beta$, Johnston et al (2013), demonstraram "in vitro" que a sinalização de IL- $\beta$ é crucial na formação experimental do AAA em ratos (38). De acordo com o autor do estudo, a IL- $\beta$ é aumentada e estimula a um estado pró-inflamatório típico do AAA e a supressão de IL- $\beta$ com o fármaco anakinra (um antagonista do receptor de interleucina 1), impede a ativação da inflamação e reduz as citocinas pró-inflamatórias, podendo ser útil como ferramenta no tratamento farmacológico. Todavia, estudos em animais de grande 
porte e ensaios clínicos controlados necessitam ser feitos para avaliar essa hipótese (39).

O Brasil ainda enfrenta dificuldades para desenvolver e implantar o serviço de Atenção Farmacêutica seja na Drogaria, nas Unidades Básicas de Saúde ou hospitais. Essas dificuldades acontecem devido ao Sistema de Saúde precário e não reconhecimento do farmacêutico como profissional indispensável nos serviços de saúde. No entanto, apesar dessas dificuldades, esse panorama está começando a mudar, pois a cada ano, vários grupos de pesquisa de brasileiros vêm realizando acompanhamento farmacoterapêutico de pacientes com hipertensão, diabetes, HIV tuberculose demonstrando que a atenção farmacêutica controla e reduz a maioria dos parâmetros clínicos da doença monitorada e problemas referentes à farmacoterapia (40-41). Todavia, ainda há poucos estudos com a Atenção Farmacêutica sobre o impacto econômico das intervenções e qualidade de vida dos pacientes. Apesar de a prática da Atenção Farmacêutica ser recente no Brasil e apresentar vários resultados positivos para o paciente e aos serviços de saúde, não há dados até o presente momento sobre a Atenção Farmacêutica em pacientes com AAA no PubMed, Science Direct e Scielo.

Deve ser ressaltado que o controle dos fatores de risco é crucial para evitar o desenvolvimento e ruptura do aneurisma e o conhecimento sobre as principais interações medicamentosas é imperativo no tratamento farmacológico do AAA. No entanto, o fornecimento de orientações corretas sobre o uso dos medicamentos e suas interações na maioria das vezes não é feito pelo profissional de saúde. Os pacientes com AAA devem ser orientados a tomar o propranolol após a refeição, pois há um aumento da biodisponibilidade do mesmo. A losartana não deve ser utilizada junto com suco de toranja, pois o mesmo inibe o principal metabólito (E3174) devido à inibição do CYP3A4 e os níveis de potássio devem ser monitorados em pacientes com insuficiência cardíaca e/ ou renal; e diabéticos devido ao risco de hipercalemia. O captopril deve ser utilizado uma hora antes ou duas horas após as refeições, pois os alimentos reduzem sua biodisponibilidade e uso de diuréticos poupadores de potássio associados aos IECA podem produzir hipercalemia se não houver um diurético de alça ou tiazídico prescrito em idosos. Há risco de hipercalemia em uso de AINE e IECA devido a deterioração da função renal e pode ocorrer redução dos efeitos anti-hipertensivos por influência na síntese de prostaglandinas. A ticlopidina deve ser tomada após as refeições, todavia, não deve ser administrada concomitantemente com ácido acetilsalicílico, varfarina e outros AINE devido ao risco aumentado de sangramento.

A sinvastatina deve ser tomada sempre à noite, pois a síntese do colesterol ocorre à noite. O risco de rabdomiólise pode ser aumentado pelo uso de sinvastatina e fármacos inibidores do CYP3A4 (ciclosporina, itraconazol, cetoconazol, eritromicina, claritromicina e inibidores de protease). As interleucinas IL-6 e IL- $\beta$ estão envolvidas na fisiopatologia do AAA e podem ser futuros alvos para o tratamento farmacológico do AAA.

\section{CONCLUSÃO}

O tratamento farmacológico do AAA é fundamental no controle dos principais fatores de risco que contribuem para o desenvolvimento e ruptura dos aneurismas pequenos. No entanto, sabendo que pacientes idosos apresentam várias limitações fisiológicas que afetam a farmacocinética dos fármacos e fazem uso vários medicamentos contribuindo para o desenvolvimento de interações medicamentosas, torna-se importante conhecer a farmacoterapia e as interações medicamentosas em pacientes com AAA. No momento da dispensação dos medicamentos o farmacêutico é o profissional mais qualificado para fornecer as informações mais relevantes sobre os medicamentos abordando o uso correto e as interações medicamentosas, e dessa forma, este profissional cria um elo de confiança com paciente.

Vários estudos da literatura vêm mostrando que a Atenção Farmacêutica em pacientes com doenças crônicas reduz os custos para o Sistema de Saúde. O foco da Atenção Farmacêutica baseia-se no acompanhamento farmacoterapêutico dos pacientes, buscando a obtenção de resultados terapêuticos desejados por meio da resolução dos problemas apresentados pelo paciente, sendo esse crucial para a atividade clínica do farmacêutico. No entanto, o Brasil ainda enfrenta algumas dificuldades para implantar o Serviço de Atenção Farmacêutica, principalmente em hospitais, unidades básicas de saúde e Drogarias, diferente de outros pases desenvolvidos como os Estados Unidos, Canadá, Espanha, Alemanha que encontraram menores dificuldades para implantar a Atenção Farmacêutica, pois além de possuírem um serviço de saúde estruturado, reconhecem o farmacêutico como profissional indispensável na área da saúde. Esse grupo de pacientes (AAA) abre portas para a Atenção Farmacêutica, pois necessita ser monitorado frequentemente pelo farmacêutico. 

25. Zaidenstein R, Soback S, Gips M, Avni B, Dishi V, Weissgarten Y, Scapa E. Effect of grapefruit juice on the pharmacokinetics of losartan and its active metabolite E3174 in healthy volunteers. Ther. Drug Monit. 2001; 23(4):45699.

26. Ferreira SH. A bradykinin-potentiating factor (BPF) present in the venom of Bothrops jararaca. Br J Pharmacol. 1965; 24(1):163-167. DOI: 10.1111/j.1476-5381.1965. tb02091.x.

27. Ferreira SH, Greene LJ Alabaster, VA, Bakhle, YS, Vane JR. Activity of various fractions of bradykinin potentiating factor against angiotensin I convertin enzyme. Nature. 1970. 225(1):379-383. DOI: 10.1038/225379a0.

28. ALLHAT. The ALLHAT Officers and Coordinators for the ALLHAT Collaborative Research Group. Major outcome in high-risk hypertensive patients to angiotensin-converting enzyme inhibitor or calcium channel blocker vs. diuretic. The Antihypertensive and LipidLowering Treatment to Prevent Heart Attack Trial (ALLHAT). JAMA. 2002; 228(23):2981-2997. DOI: 10.1001/ archinte.165.8.936

29. Lourenço R. Enteral feeding: drug/nutrient interaction. Clin Nutr. 2001; 20(2): 187-193. DOI: 10.1054/ clnu. 2000.0155

30. Sica DA, Gehr TWB. Angiotensin-converting enzyme inhibitors. In: Oparil S, Weber MA (ed). Hypertension. Philadelphia, Pennsylvania: Elsevier Saunders. 2005. p. 669-682.

31. Gus M. Farmacologia clínica dos medicamentos anti-hipertensivos. In: Brandão AA, Amodeo C, Nobre F, Fuchs FD . Hipertensão. Rio de Janeiro: Elsevier; 2006; p. 326335.

32. MEDLEY. Disponível em htt:/www.medley.com.br/ portal/bula/cloridrato_de_ticlopidina_comprimidos.pdf, acessado em 13/2/2015.

33. Reinhardt SC, Vaughan CJ. Statins in acute coronary syndromes. Drugs Today. 2002; 38(5):339-350. DOI: 10.1002/14651858.CD006870.pub2.
34. Ferreira WP, Bertolami MC, Santos SN, Barros MRA, Barretto RB, Pontes Jr SC, Carvalho AC. One-month therapy simvastatin restores endothelial function in hypercholesterolemic children and adolescents. Pediatr Cardiol. 2007; 28(1): 8-13. DOI: 10.1007/s00246-0051304-x.

35. Pereira EC, Bertolami MC, Faludi AA, Sevanian A, Abdalla DS. Antioxidant effect of simvastatin is not enhanced by its association with alphatocopherol in hypercholesterolemic patients. Radic Biol Med. 2004; 37(9):1440-1448. DOI: 10.1016/j.freeradbiomed.2004.07.019.

36. Magalhães CME. Mecanismos de rabdomiólise com as estatinas. Arq Bras Cardiol. 2005; 85(Suppl-5):1-6.

37. Sugimoto KI, Ohmori M, Tsuruoka S, Nishiki K, Kawaguchi, A, Harada, KI, Fujimura A. Different effects of St John's Wort on the pharmacokinetics of simvastatin and pravastatin. Clin Pharmacol Ther. 2001; 70(6): 518-524. DOI: 10.1016/S0009-9236(01)64092-X.

38. Johnston WF, Salmon M, Su G, Lu G, Stone ML, Zhao Y, Ailawadi G. Genetic and pharmacologic disruption of interleukin-1 $\beta$ signaling inhibits experimental aortic aneurysm formation. Arterioscler. Thromb. Vasc. Biol. 2013; 33(2): 294-304. DOI: 0.1161.ATVBA.112.300432.

39. Harrison SC, Smith AJ, Jones GT, Swerdlow DI, Rampuri R, Bown MJ, Humphries SE. Interleukin-6 receptor pathways in abdominal aortic aneurysm. Eur Heart J. 2013; 34(48): 3707-3716. DOI: 10.1093/eurheartj/ehs354

40. Stein A, IS Mastroianni PC. Outcomes of pharmaceutical care in Brazil: a literature review. Rev Ciênc Farm Básica Apl. 2013; 34(4):475-480.

41. Obreli-Neto PR, Marusic S, Guidoni CM, Baldoni Ade O, Renovato RD, Pilger D, Cuman RK, Pereira LR. Economic Evaluation of a Pharmaceutical Care Program for Elderly Diabetic and Hypertensive Patients in Primary Health Care: A 36-Month Randomized Controlled Clinical Trial. J. Manag. Care Pharm. 2015; 21(1): 66-75. 